\title{
(6) OPEN ACCESS \\ Extending the diabetic retinopathy screening interval beyond 1 year: systematic review
}

\author{
Sian Taylor-Phillips, Hema Mistry, Rachael Leslie, Dan Todkill, Alexander Tsertsvadze, \\ Martin Connock, Aileen Clarke
}

- Additional material is published online only. To view please visit the journal online (http://dx.doi.org/10.1136/ bjophthalmol-2014-305938).

Warwick Medical School, The University of Warwick, Coventry, UK

\section{Correspondence to} Professor Aileen Clarke Warwick Medical School, The University of Warwick, Coventry, CV4 7AL, UK; aileen.clarke@warwick.ac.uk

Received 2 August 2014 Revised 21 November 2014 Accepted 14 December 2014 Published Online First 13 January 2015
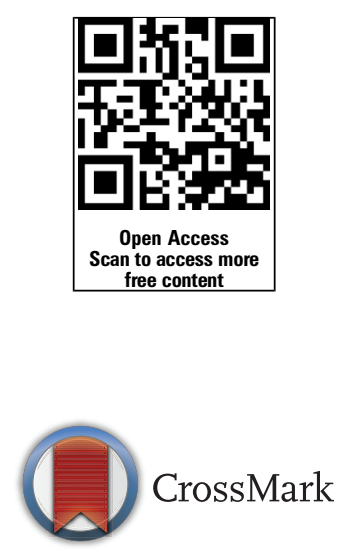

\footnotetext{
To cite: Taylor-Phillips $S$, Mistry $\mathrm{H}$, Leslie $\mathrm{R}$, et al. $\mathrm{Br} \mathrm{J}$ Ophthalmol 2016;100: 105-114.
}

\section{ABSTRACT}

To determine whether the recommended screening interval for diabetic retinopathy (DR) in the UK can safely be extended beyond 1 year. Systematic review of clinical and cost-effectiveness studies. Nine databases were searched with no date restrictions. Randomised controlled trials (RCTs), cohort studies, prognostic or economic modelling studies which described the incidence and progression of DR in populations with type 1 diabetes mellitus or type 2 diabetes mellitus of either sex and of any age reporting incidence and progression of DR in relation to screening interval (vs annual screening interval) and/or prognostic factors were included. Narrative synthesis was undertaken. 14013 papers were identified, of which 11 observational studies, 5 risk stratification modelling studies and 9 economic studies were included. Data were available for 262541 patients of whom at least $228649(87 \%)$ had type 2 diabetes. There were no RCTs. Studies concluded that there is little difference between clinical outcomes from screening 1 yearly or 2 yearly in low-risk patients. However there was high loss to follow-up (13-31\%), heterogeneity in definitions of low risk and variation in screening and grading protocols for prior retinopathy results. Observational and economic modelling studies in low-risk patients show little difference in clinical outcomes between 1-year and 2-year screening intervals. The lack of experimental research designs and heterogeneity in definition of low risk considerably limits the reliability and validity of this conclusion. Costeffectiveness findings were mixed. There is insufficient evidence to recommend a move to extend the screening interval beyond 1 year.

\section{INTRODUCTION}

Diabetic retinopathy (DR) is a serious complication of diabetes mellitus and a major cause of visual loss globally. ${ }^{12}$ Prevalence of DR is rising. ${ }^{2}$ Early detection and timely treatment of sight-threatening DR have reduced the incidence and progression of visual loss. ${ }^{3-8}$ Screening for DR (using ophthalmoscopy and fundus photography) is accurate, safe and cost-effective. ${ }^{9-11}$

DR screening is recommended in many countries. ${ }^{12-16}$ However there is often no complete register of patients and non-uniformity of interval, coverage, uptake, screening methods and grading. ${ }^{17} 18$ The generalisablity of findings from one country to another has been questioned. ${ }^{19}$ There has been considerable international debate about extending the screening interval in the UK and the USA. A previous systematic review ${ }^{20}$ advocated a longer screening interval as a cost saving measure with various caveats but the authors did not formally critically appraise the evidence base, and two studies have since been published.

This systematic review aims to investigate the effects of longer screening intervals (vs an annual screening interval) in people with diabetes in order to inform screening decisions in the UK.

\section{METHODS}

\section{Study eligibility criteria}

Randomised controlled trials (RCTs), cohort studies, prognostic or economic modelling studies which described the incidence and progression of DR in populations with type 1 diabetes mellitus (T1DM) or type 2 diabetes mellitus (T2DM) of either sex and of any age investigating annual with longer intervals were eligible (articles where annual screening is compared with a shorter interval were also included, because for some patient groups this interval may have to be more frequent). Studies investigating DR but not related to screening intervals, full text non-English publications, editorials, letters or commentaries were excluded.

\section{Search strategy}

Electronic searches were performed on 1 October 2013 in nine databases including Medline, SCOPUS, EMBASE, Cochrane Database of Systematic Reviews, Cochrane Central Register of Controlled Trials, Cochrane Methodology Register, Database of Abstracts of Reviews of Effects, Health Technology Assessment Database, and the National Health Service (NHS) Economic Evaluation Database. No date or language restrictions were applied. Search terms were left deliberately broad. Reference lists of relevant systematic reviews were checked. Online supplementary appendix 1 gives details of the search strategy.

\section{Study selection}

Abstracts from the different databases were merged and duplicates removed. Two reviewers independently screened abstracts using prepiloted eligibility criteria and also independently reviewed the identified full texts. Differences were discussed and agreed with the input of a third adjudicator where necessary.

\section{Data extraction and assessment of methodological quality}

Two independent reviewers extracted data including: study characteristics, population characteristics, screening data, postscreening data, outcome measures and conclusions. Economic appraisals data were extracted and assessed using Consolidated Health Economic Evaluation Reporting Standards statement ${ }^{21}$ and an adapted checklist for economic 
models was also used. ${ }^{22}$ Observational studies were assessed using the Critical Appraisal Skills Programme tool. ${ }^{23}$ Risk prediction studies were evaluated, but focused on external validation and impact on outcomes. ${ }^{24}$ Disagreements were resolved through discussion with a third reviewer.

\section{Data synthesis and analysis}

Evidence was synthesised narratively, because of heterogeneity in populations, screening intervals, measurement methods, outcomes and uptake. Evidence on incidence and/or progression of DR was synthesised separately in relation to (A) screening intervals and (B) risk factors.

\section{RESULTS}

Fourteen thousand and thirteen records were identified after duplicates were removed and 142 were screened at full text level. Of these 113 were excluded, leaving 29 eligible records, reporting on 26 unique studies (figure 1). ${ }^{1} 6925-47$ One study was reported in two publications: one reporting prevalence of $\mathrm{DR}$ in relation to screening intervals ${ }^{25}$ and the other incidence and progression of DR in relation to prognostic factors. ${ }^{26}$ A study by Mehlsen et al was reported in two publications. ${ }^{27} 28$ Analyses from two other publications were based on the same study population. ${ }^{29} 30$ To avoid a potential for double-counting, the abovementioned six publications were considered as three

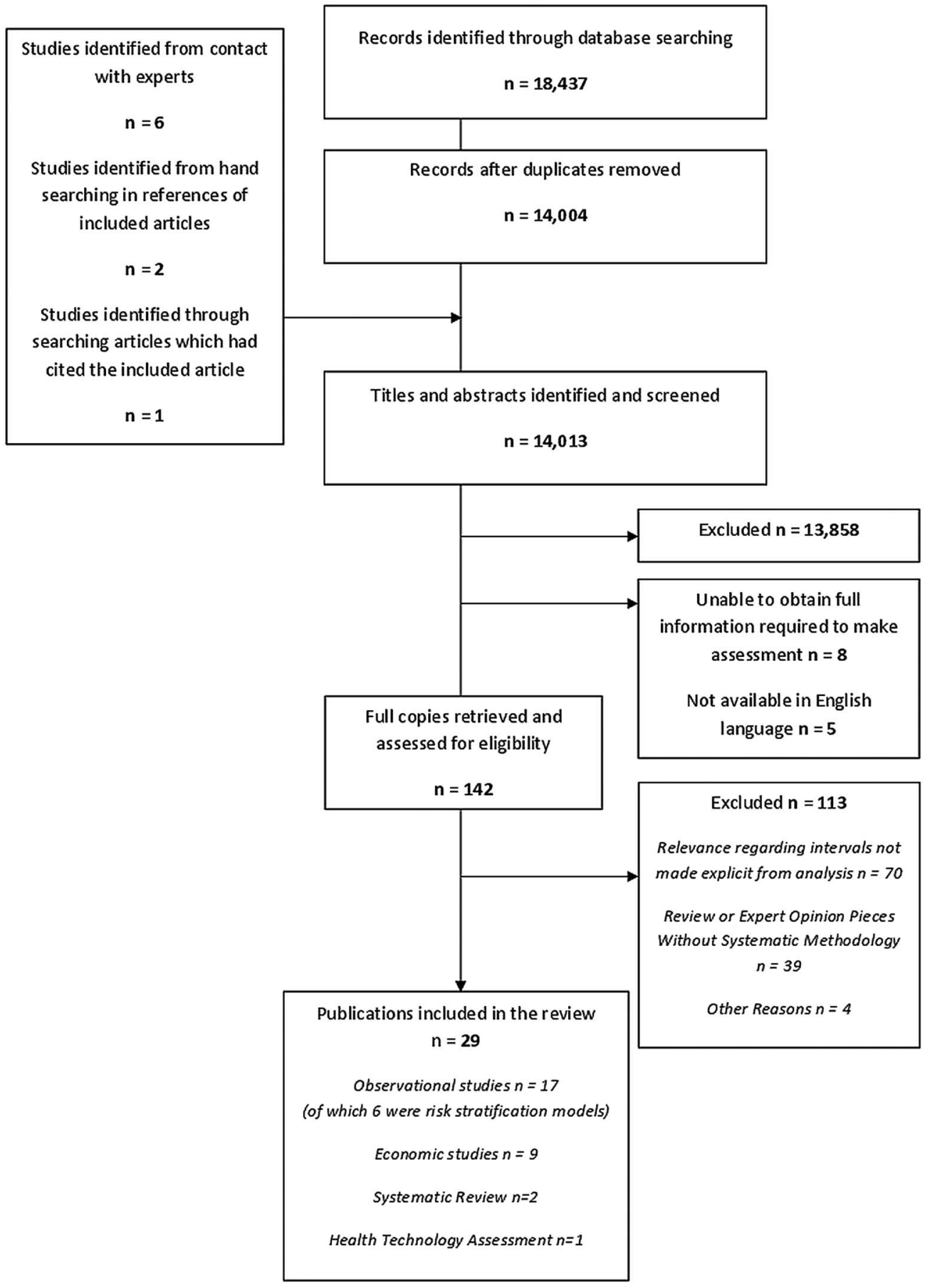

Figure 1 PRISMA flow chart of systematic review. 
unique studies. One additional study was identified from searching of reference lists. ${ }^{31}$

\section{Incidence and progression of DR}

Incidence and/or progression of DR was reported in 15 studies. ${ }^{1} 25-40$ There were no randomised or non-randomised comparative studies. Ten observational studies followed progression of retinopathy in single populations with defined screening

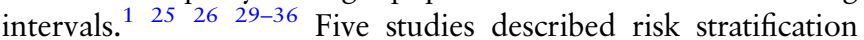
algorithms. None were externally validated. ${ }^{27} 28$ 37-40

Most observational studies used annual screening proto$\operatorname{cols}^{25} 26 \quad 34-36$ although actual screening intervals varied. Two studies described screening every 3 years ${ }^{31} 33$ and one every 2 years. $^{30}$ Populations studied were from UK, ${ }^{1} \begin{array}{lllll}25 & 26 & 33 & 35 & 36\end{array}$ Iceland, ${ }^{29}{ }^{30}$ Australia $^{34}$ and Sweden. ${ }^{32}$ Different strategies for grading retinopathy were employed (see table 1 ).

The Critical Appraisal Skills Programme tool (see online supplementary appendix 2) indicated that observational studies reported well. Completeness of follow-up varied, with attrition reaching up to $31 \%$ in two studies. ${ }^{25} 36$ Three studies had lower losses to follow-up (under $25 \% \%^{32} 2935$ ), and reported that baseline characteristics did not differ between responders/completers and those lost to follow-up. In one study ${ }^{1}$ for which the rate of non-attendance for a repeat screen was relatively low (13\%), the authors reported that non-attendees tended to be older with a longer duration of diabetes in comparison with attendees. Completeness of follow-up data was not reported for four studies. $^{26} 303334$

Incidence and/or progression of DR in relation to screening intervals was reported in six observational studies (see online supplementary appendix 3). ${ }^{25} 26 \quad 28 \quad 34-37 \quad 40$ All six studies suggested that the annual screening interval for patients with T1DM/T2DM at low risk (ie, no visible DR at baseline, adequate diabetes control) could be safely extended to 2 years or beyond. In three studies the incidence and progression of DR, either actual or predicted, were similar between annual and 2-yearly screening. ${ }^{25} 28{ }^{34}$ Younis et $a l,{ }^{35} 36$ found that about $95 \%$ of patients with T1DM-T2DM with no DR at initial screening remained free of sight threatening diabetic retinopathy (STDR) for the mean screening interval of 5.4 years. In a large single arm study in more than 20000 patients, no significant association between a longer screening interval (18-24 months vs $12-18$ months) and prevalence of referable $\mathrm{DR}(\mathrm{OR}=0.93$, $95 \%$ CI 0.82 to 1.05$)$ or STDR/maculopathy $(\mathrm{OR}=1.05,95 \%$ CI 0.72 to 1.52 ) was found. ${ }^{25}$ In the largest study, of 155000 patients, ${ }^{40}$ predicted risk of progression in patients with T2DM with no DR at two consecutive screens for the 2-year screening interval was $0.15 \%$ or less compared with $0.03 \%$ or less for the annual interval. Studies which investigated 2-year screening intervals for patient subgroups at increased risk of progression (eg, visible DR of any grade, poorly controlled diabetes, duration of DM >10 years), suggested that these subgroups may need to be screened more frequently.

Incidence and/or progression of DR in relation to prognostic factors were reported in 13 studies (see online supplementary appendix 3). ${ }^{125-27} 29-3638-40$ Most studies showed that patients with baseline DR (vs no DR) were at higher risk of progressing to referable DR or STDR. ${ }^{26} 33 \quad 35-37 \quad 3940$ In one study of patients with T2DM, ${ }^{26}$ the presence of baseline background DR (vs no background DR) was associated with an increased risk of pre-DR or proliferative DR $(\mathrm{HR}=5.00,95 \%$ CI 4.40 to 5.60$)$. Mehlsen et al, ${ }^{27}$ also found the number of retinal haemorrhages to be a significant risk factor for progression to STDR in patients with $\mathrm{T} 1 \mathrm{DM}(\mathrm{OR}=2.68,95 \% \mathrm{CI} 1.83$ to 3.91$)$ and
T2DM (OR $=2.37,95 \%$ CI 1.84 to 3.06). Grade of baseline DR was also a significant predictor of progression to referable DR or STDR. ${ }^{33}$ 35-37 Clinical risk factors for progression to STDR were found to be longer with duration of diabetes, ${ }^{1} 26273435373840$ insulin use, ${ }^{1263638}$ higher level of HbA1c, ${ }^{27} 3034 \quad 3738$ raised systolic blood pressure ${ }^{37} 38$ and hypertension treatment. ${ }^{26}$

Six studies ${ }^{27} \quad 28 \quad 37-40$ describe risk stratification models to determine the most effective screening interval based on identified risk factors. These were developed using either Cox proportional hazards models, non-parametric survival models or logistic regression with data from different screening programmes. ${ }^{27} 28 \quad 37-40$ None of the models were externally validated on a UK data set, although one model $^{37}$ was externally validated on a Danish database. All other models were internally validated tending to overestimate performance in comparison with external validation, due to statistical overfitting and potential lack of generalisability. ${ }^{48}$ The Danish screening programme model $^{27} 28$ indicated that screening could be prolonged 2.9 times for patients with T1DM and 1.2 times for patients with T2DM without increasing STDR. The Aspelund risk prediction algorithm ${ }^{37}$ when externally validated on the same Danish data set could reduce the number of visits by $59 \%$ without increasing overall risk using the predictors of disease type (1 or 2 ), HbA1c, systolic blood pressure, gender and presence of non-proliferative DR. The current Danish screening system has screening intervals dependent on risk factors, so generalisability to the UK is problematic. Risk stratification based on two consecutive negative screens had the potential to reduce the number of screening visits in the Scottish screening service by up to $40 \%{ }^{40}$ However, probability of referable retinopathy would be higher with a 2 year interval, increasing from $0.7 \%$ to $1.2 \%$ in the English model (where screening includes mydriasis and two fields per eye) ${ }^{39}$ and from $0.05 \%$ to $0.25 \%$ in men with 10 years duration of diabetes in the Scottish model (where screening includes one field per eye). ${ }^{40}$ These rates were higher when any referable disease was included.

\section{Economic evaluation of screening intervals}

Nine papers assessed cost-effectiveness of differing screening intervals for identification of DR/prevention of DR complications. ${ }^{6}{ }^{9} 41-47$ Studies used various types of simulation models conducted on hypothetical cohorts of patients with diabetes using data from existing data sets and literature.

Table 2 highlights key characteristics, methods and findings. Not all studies reported a viewpoint. The most common outcomes were sight years saved/gained, and two studies used quality-adjusted life years. ${ }^{46} 47$ Two studies did not discount. ${ }^{42} 44$ Four studies did not provide an incremental cost-effectiveness ratio.

Most items in the Consolidated Health Economic Evaluation Reporting Standards checklist ${ }^{21}$ were well reported (see online supplementary appendix 4). Using the Phillips ${ }^{24}$ checklist, publications reported a clear statement of the decision problem, objectives, data sources, model, methods and assumptions. They also reported costs including sources, and most also adequately compared results with previous models (see online supplementary appendix 5).

In general, the cost-effectiveness studies suggested that screening every 2 years could be safely adopted for those with no background or mild retinopathy, ${ }^{6} 424647$ without increased risk of visual loss, and this reduces screening costs by $\sim 25 \%,{ }^{42}$ and has no detrimental effect on years of sight saved. ${ }^{6}$ When taking into account the ability to detect other eye conditions screening 
Table 1 Differences in screening and grading protocols for detecting diabetic retinopathy

\begin{tabular}{|c|c|c|c|c|c|c|c|c|c|}
\hline & $\begin{array}{l}\text { Was mydriasis } \\
\text { used? }\end{array}$ & $\begin{array}{l}\text { How many and which } \\
\text { retinal fields were } \\
\text { taken? }\end{array}$ & $\begin{array}{l}\text { Photographs or } \\
\text { digital retinal } \\
\text { photographs }\end{array}$ & $\begin{array}{l}\text { Which cameras } \\
\text { were used? }\end{array}$ & $\begin{array}{l}\text { Were patients tested } \\
\text { using slit lamp } \\
\text { (biomicroscopy) }\end{array}$ & $\begin{array}{l}\text { What grading protocol } \\
\text { was used? }\end{array}$ & $\begin{array}{l}\text { Were screeners and } \\
\text { graders trained and/or } \\
\text { accredited? }\end{array}$ & $\begin{array}{l}\text { Was grading quality } \\
\text { assured?/ Was grading } \\
\text { assessed elsewhere? }\end{array}$ & $\begin{array}{l}\text { How many } \\
\text { times were } \\
\text { images } \\
\text { graded? }\end{array}$ \\
\hline $\begin{array}{l}\text { Agardh and } \\
\text { Tababat-Khani }^{22}\end{array}$ & No information & $\begin{array}{l}\text { One central and one } \\
\text { nasal } 50^{\circ} \text { field per eye. }\end{array}$ & $\begin{array}{l}\text { Red free digital } \\
\text { images }\end{array}$ & No information & No information & $\begin{array}{l}\text { International Diabetic } \\
\text { Retinopathy and Macula } \\
\text { Edema Severity Scales }\end{array}$ & $\begin{array}{l}\text { Performed by specially } \\
\text { trained ophthalmic } \\
\text { nurses }\end{array}$ & No information & No information \\
\hline Jones et a $P^{26}$ & $\begin{array}{l}\text { Both pupils were } \\
\text { dilated with } 1 \% \\
\text { tropicamide drops }\end{array}$ & $\begin{array}{l}\text { Two photographs of } \\
\text { each eye were taken, } \\
\text { one centred on the optic } \\
\text { nerve and the other on } \\
\text { the fovea. } \\
\text { Images taken by trained } \\
\text { retinal screeners }\end{array}$ & $\begin{array}{l}\text { Mixed } \\
\text { Before 2000: colour } \\
\text { transparency film } \\
\text { From 2000: digital } \\
\text { imaging }\end{array}$ & $\begin{array}{l}\text { Mobile retinal } \\
\text { cameras: Canon } \\
45 \mathrm{NM} \text { or } 46 \mathrm{NM} \\
\text { fundus cameras } \\
\text { (Canon UK, Reigate, } \\
\text { UK) with } 458 \text { fields } \\
\text { and Orion Eyecap and } \\
\text { DRSS digital imaging } \\
\text { software. }\end{array}$ & No information & $\begin{array}{l}\text { 1990-2002: Descriptive } \\
\text { grading system based on } \\
\text { European guidelines } \\
\text { From 2003: ..K. National } \\
\text { Screening Committee } \\
\text { grading system } \\
\text { After 2006: NSC grading } \\
\text { system } \\
\text { Described as 'virtually } \\
\text { identical' }\end{array}$ & $\begin{array}{l}\text { Before 2000: } \\
\text { diabetologist with a } \\
\text { specialist interest in } \\
\text { retinopathy } \\
\text { From 2000: seven } \\
\text { primary graders }\end{array}$ & $\begin{array}{l}\text { Yes. Nationally accredited } \\
\text { arbitration grader }\end{array}$ & No information \\
\hline Kohner et $\left.a\right|^{33}$ & Yes & $\begin{array}{l}\text { Four-field } 30^{\circ} \text { retinal } \\
\text { photographs taken as } \\
\text { stereo pairs }\end{array}$ & No information & No information & No information & $\begin{array}{l}\text { Allocated to a } \\
\text { retinopathy severity level } \\
\text { using the Early Treatment } \\
\text { of Diabetic Retinopathy } \\
\text { Study (ETDRS) final scale, } \\
\text { modified for four } \\
\text { standard fields. } \\
\text { Retinopathy severity } \\
\text { categorised as no } \\
\text { retinopathy, MA only in } \\
\text { one eye, MA in both eyes } \\
\text { or more severe } \\
\text { retinopathy features. }\end{array}$ & No information & $\begin{array}{l}\text { Only patients with a set } \\
\text { of good quality images of } \\
\text { both eyes were included } \\
\text { in the study. }\end{array}$ & No information \\
\hline $\begin{array}{l}\text { Kristinsson } \\
\text { et a }\left.\right|^{29}\end{array}$ & Yes & No information & No information & No information & Yes & No information & No information & No information & No information \\
\hline Looker et $a l^{40}$ & If required & Single field & Digital photograph & No information & $\begin{array}{l}\text { Slit lamp outcomes were } \\
\text { not available for all } \\
\text { patients, but where } \\
\text { available, results were } \\
\text { used. }\end{array}$ & Scottish grading system & No information & No information & No information \\
\hline Maguire et a ${ }^{\beta 4}$ & $\begin{array}{l}\text { Yes-1\% } \\
\text { cyclopentolate } \\
\text { and 2.5\% } \\
\text { phenylephrine }\end{array}$ & $\begin{array}{l}\text { Stereoscopic fundal } \\
\text { photography of seven } \\
\text { fields. Non-simultaneous } \\
\text { photographic pairs for } \\
\text { each eye }\end{array}$ & $\begin{array}{l}\text { Viewed with a } \\
\text { Donaldson } \\
\text { Stereoviewer } \\
\text { providing a 3D } \\
\text { representation of } \\
\text { the fundus. }\end{array}$ & $\begin{array}{l}\text { Topcon fundus } \\
\text { camera }\end{array}$ & $\begin{array}{l}\text { Yes. Slit lamp } \\
\text { examination of the } \\
\text { anterior segment. }\end{array}$ & $\begin{array}{l}\text { ETDRS adaption of the } \\
\text { modified Ailie House } \\
\text { classification of diabetic } \\
\text { retinopathy. }\end{array}$ & $\begin{array}{l}\text { Graded by an } \\
\text { ophthalmologist with a } \\
\text { large sample graded by } \\
\text { a second grader } \\
\text { independently. }\end{array}$ & $\begin{array}{l}\text { When necessary, a } \\
\text { grading supervisor was } \\
\text { used to adjudicate. } \\
\text { Agreement between two } \\
\text { graders was statistically } \\
\text { assessed. }\end{array}$ & No information \\
\hline Misra et $a^{25}$ & As Jones et al & & & & & & & & \\
\hline $\begin{array}{l}\text { Olafsdótttir and } \\
\text { Stefánsson }\end{array}$ & Yes & & $\begin{array}{l}\text { Colour photographs } \\
\text { taken with a } \\
\text { 90-diopter lens }\end{array}$ & & Yes & $\begin{array}{l}\text { Visual acuity reported by } \\
\text { the better eye. } \\
\text { Retinopathy level } \\
\text { determined as the stage } \\
\text { of the worse eye. } \\
\text { Visual acuity measured }\end{array}$ & $\begin{array}{l}\text { Screened by an } \\
\text { ophthalmologist }\end{array}$ & & \\
\hline
\end{tabular}


Table 1 Continued

\begin{tabular}{|c|c|c|c|c|c|c|c|c|c|}
\hline & $\begin{array}{l}\text { Was mydriasis } \\
\text { used? }\end{array}$ & $\begin{array}{l}\text { How many and which } \\
\text { retinal fields were } \\
\text { taken? }\end{array}$ & $\begin{array}{l}\text { Photographs or } \\
\text { digital retinal } \\
\text { photographs }\end{array}$ & $\begin{array}{l}\text { Which cameras } \\
\text { were used? }\end{array}$ & $\begin{array}{l}\text { Were patients tested } \\
\text { using slit lamp } \\
\text { (biomicroscopy) }\end{array}$ & $\begin{array}{l}\text { What grading protocol } \\
\text { was used? }\end{array}$ & $\begin{array}{l}\text { Were screeners and } \\
\text { graders trained and/or } \\
\text { accredited? }\end{array}$ & $\begin{array}{l}\text { Was grading quality } \\
\text { assured?/ Was grading } \\
\text { assessed elsewhere? }\end{array}$ & $\begin{array}{l}\text { How many } \\
\text { times were } \\
\text { images } \\
\text { graded? }\end{array}$ \\
\hline & & & & & & $\begin{array}{l}\text { on a Snellen chart at } 6 \mathrm{~m} \\
\text { with the best refractive } \\
\text { correction }\end{array}$ & & & \\
\hline $\begin{array}{l}\text { Soto-Pedre } \\
\text { et } a^{\beta 1}\end{array}$ & No Information & $\begin{array}{l}\text { One fundus photograph } \\
\text { centred on the macula } \\
\text { of each eye taken with } \\
45^{\circ} \text { non-mydriatic retinal } \\
\text { camera }\end{array}$ & Instant film Polaroid & Canon CR4-45NM & No & $\begin{array}{l}\text { International Diabetic } \\
\text { Retinopathy and Macula } \\
\text { Edema Severity Scales. } \\
\text { Level of disease recorded } \\
\text { for the worse eye. }\end{array}$ & $\begin{array}{l}\text { Stored polaroid } \\
\text { photographs were } \\
\text { graded by the same } \\
\text { retina specialist for this } \\
\text { study. }\end{array}$ & No & $\begin{array}{l}\text { Once for the } \\
\text { purpose of this } \\
\text { retrospective } \\
\text { study }\end{array}$ \\
\hline Stratton et $a P^{\beta 9}$ & Yes & $\begin{array}{l}\text { Two standard } 45 \text { fields } \\
\text { — Macular and disc } \\
\text { centred—per eye }\end{array}$ & $\begin{array}{l}\text { Digital colour } \\
\text { retinal photographs }\end{array}$ & No information & No information & $\begin{array}{l}\text { Grading based on the } \\
\text { ETDRS severity scale } \\
\text { Background retinopathy } \\
\text { defined using the R1M0 } \\
\text { category on the English } \\
\text { NHS Diabetic Eye } \\
\text { Screening Programme. }\end{array}$ & $\begin{array}{l}\text { Trained assessors in a } \\
\text { central location to the } \\
\text { screening venues }\end{array}$ & $\begin{array}{l}\text { Internal and external } \\
\text { quality-assured reading } \\
\text { process that reaches } \\
\text { national } \\
\text { recommendations. }\end{array}$ & No information \\
\hline Thomas et al ${ }^{1}$ & $\begin{array}{l}\text { Tropicamide } \\
\text { (applied to each } \\
\text { eye } 15 \text { min before } \\
\text { screening }\end{array}$ & $\begin{array}{l}\text { Two } 45^{\circ} \text { digital retinal } \\
\text { images per eye-one } \\
\text { macular centred and one } \\
\text { nasal field }\end{array}$ & $\begin{array}{l}\text { Non-mydriatic } \\
\text { Canon DGi camera }\end{array}$ & & & $\begin{array}{l}\text { Screening undertaken by } \\
\text { a trained photographer } \\
\text { Grading undertaken by } \\
\text { trained staff use an } \\
\text { enriched version of } \\
\text { English National } \\
\text { Screening Protocol }\end{array}$ & $\begin{array}{l}\text { Before screening, a } \\
\text { trained healthcare } \\
\text { assistant assesses visual } \\
\text { acuity in both eyes } \\
\text { using an illuminated } \\
3 \mathrm{~m} \text { Snellen chart }\end{array}$ & & $\begin{array}{l}\text { Retinal images } \\
\text { transferred to a } \\
\text { central reading } \\
\text { centre for } \\
\text { grading }\end{array}$ \\
\hline Younis et $a l^{35}$ & $\begin{array}{l}1 \% \text { tropicamide } \\
\text { with or without } \\
\text { phenylephrine }\end{array}$ & $\begin{array}{l}\text { Three overlapping } \\
\text { non-stereoscopic } 33 \mathrm{~mm} \\
\text { transparency } \\
\text { photographs of each eye }\end{array}$ & $\begin{array}{l}\text { Either Canon } \\
\text { CR4-45NM with } \\
45^{\circ} \text { fields or a } \\
\text { Topcon TRC } 50 \text { SX } \\
\text { camera with } 50^{\circ} \\
\text { fields. }\end{array}$ & No information & $\begin{array}{l}\text { Patients with } \\
\text { ungradable images or } \\
\text { STDR invited for slit } \\
\text { lamp biomicroscopy by } \\
\text { specialists in medical } \\
\text { retinal disease. }\end{array}$ & $\begin{array}{l}\text { STDR defined as } \\
\text { moderate preproliferative } \\
\text { retinopathy or greater } \\
\text { and/or significant } \\
\text { maculopathy in any eye. } \\
\text { Graded by trained } \\
\text { graders with a modified } \\
\text { Wisconsin algorithm. }\end{array}$ & No information & No information & No information \\
\hline Younis et $\left.a\right|^{36}$ & As Younis 2003b & & & & & & & & \\
\hline
\end{tabular}

NSC, National Screening Committee; MA, microaneurysms; STDR, sight threatening diabetic retinopathy. 


\begin{tabular}{|c|c|c|c|}
\hline $\begin{array}{l}\text { Author } \\
\text { (year) }\end{array}$ & $\begin{array}{l}\text { Type of economic } \\
\text { evaluation and model }\end{array}$ & Population studied & Comparators \\
\hline $\begin{array}{l}\text { Brailsford } \\
\text { et al } \\
(2007)^{41}\end{array}$ & $\begin{array}{l}\text { EE: CEA } \\
\text { Model: Discrete event } \\
\text { simulation embedded in an } \\
\text { optimisation model using } \\
\text { POST }\end{array}$ & $\begin{array}{l}\text { Hypothetical population of } \\
100000 \text { people with T2DM }\end{array}$ & $\begin{array}{l}\text { Two screening policies using different } \\
\text { strategies vs no screening: } \\
\text { 1. optometrist funduscopy } \\
\text { 2. diabetologist ophthalmoscopy } \\
\text { 3. GP ophthalmoscopy } \\
\text { 4. mobile camera } \\
\text { 5. mydriatic } 7 \text { field photography by } \\
\text { ophthalmologist (gold standard). } \\
\text { Screening interval was varied } \\
\text { between } 6 \text { months and } 36 \text { months. }\end{array}$ \\
\hline $\begin{array}{l}\text { Chalk et al } \\
(2012)^{42}\end{array}$ & $\begin{array}{l}\text { EE: CEA } \\
\text { Model: Simulation model } \\
\text { using POST }\end{array}$ & $\begin{array}{l}\text { Hypothetical population of } \\
5000 \text { people with T2DM } \\
\text { without DR }\end{array}$ & $\begin{array}{l}\text { Annual (or 6-monthly) screening vs a } \\
\text { 2-year screening programme }\end{array}$ \\
\hline
\end{tabular}

\begin{tabular}{|c|c|c|c|}
\hline $\begin{array}{l}\text { Dasbach } \\
\text { et al } \\
(1991)^{43}\end{array}$ & $\begin{array}{l}\text { EE: CEA } \\
\text { Model: Simulation model } \\
\text { using a Markov process }\end{array}$ & $\begin{array}{l}\text { Hypothetical groups of a } \\
1000 \text { patients with onset } \\
\text { diabetes: } \\
\text { 1. younger patients; } \\
\text { 2. older patients taking } \\
\text { insulin; and } \\
\text { 3. older patients, not taking } \\
\text { insulin }\end{array}$ & $\begin{array}{l}\text { Seven screening strategies: } \\
\text { (1) no care } \\
\text { (2) and (3) annual or biannual visits } \\
\text { to a community healthcare } \\
\text { professional } \\
\text { (4) and (5) annual or biannual } \\
\text { non-mydriatic camera screening } \\
\text { (6) and (7) annual or biannual } \\
\text { mydriatic camera screening }\end{array}$ \\
\hline $\begin{array}{l}\text { Davies et al } \\
(2002)^{44}\end{array}$ & $\begin{array}{l}\text { EE: CEA } \\
\text { Model: Discrete event } \\
\text { simulation model using } \\
\text { POST. }\end{array}$ & $\begin{array}{l}\text { Hypothetical population of } \\
500000 \text { people with T1DM } \\
\text { or T2DM who could develop } \\
\text { DR }\end{array}$ & $\begin{array}{l}\text { Each scenario compared with no } \\
\text { screening. Screening done by a mobile } \\
\text { camera, diabetologist, optometrist or } \\
\text { GP. } \\
\text { Policy } 1 \text {, screening every } 12 \text { months } \\
\text { and a } 6 \text {-month interval between visits } \\
\text { once DR detected. } \\
\text { Policy 2, screening every } 12 \text { months, } \\
\text { even after the detection of } \\
\text { background retinopathy, until } \\
\text { treatable retinopathy is detected } \\
\text { (every } 6 \text { months). } \\
\text { Mydriatic seven-field photography by } \\
\text { an ophthalmologist, screening every } \\
6 \text { months, with visits every } 3 \text { months } \\
\text { after DR had been detected. }\end{array}$ \\
\hline $\begin{array}{l}\text { Javitt et al } \\
(1990)^{45}\end{array}$ & $\begin{array}{l}\text { EE: CEA } \\
\text { Model: Monte Carlo } \\
\text { Simulation model using } \\
\text { PROPHET }\end{array}$ & $\begin{array}{l}\text { Hypothetical cohort of } \\
\text { patients with T1DM }\end{array}$ & $\begin{array}{l}\text { Five screening strategies all have } \\
\text { dilated ophthalmoscopy: } \\
\text { 1. every } 2 \text { years } \\
\text { 2. annually } \\
\text { 3. annually for patients with no DR }\end{array}$ \\
\hline
\end{tabular}

Methods (perspective, tim horizon and discount rate)

Study perspective: Not stated Time horizon: 100 years Discount rate: $0 \%, 3 \% 5 \%$ Currency/price year: UK $\mathrm{f}$-year not stated fear not stated without DR
Study perspective: Not stated Time horizon: 15 years Discount rate: Not stated Currency/price year: UK f-year not stated

Study perspective: Societal Time horizon: 10 years and 60 years

Discount rate: $5 \%$ (varied between $0 \%$ and $10 \%$ )

Currency/price year: US\$ in 1989 prices

Study perspective: Not stated Time horizon: 25 years Discount rate: Not undertaken Currency/price year: UK

$\mathrm{f}$-year not stated
Methods (costs, outcomes,

ICER and sensitivity

analyses)

Outcomes: Total number of years of sight saved

Costs: Direct costs of screening and treatment, outpatient visits ICER: Incremental cost per year of sight saved

Sensitivity analyses: Not stated

Outcomes: Proportion of patients with diabetes with patients with
vision loss

vision loss
Costs: Screening test,

ophthalmology visits and lase treatment

ICER: None stated

Sensitivity analyses: One-way

Outcomes: Sight years saved

Costs: Screening and clinic

visits, treatments and

rehabilitation

ICER: None stated

Sensitivity analyses: One-way

Outcomes: Average years of sight saved

Costs: Screener, ophthalmolog outpatient visits, treatment and mobile camera (including set-up costs).

ICER: Costs per year of sight

Sensitivity analyses: One-way

Results and main conclusions

Most cost-effective screening policy is where the optometrist carries out both screens (policy 2) the gold stand 2 is post $S$ creening should be carried out at 30 month intervals.

The 2-year screening costs were f1 360516 and annual screening costs were $f 1834060$, which represents a $25.8 \%$ reduction in screening costs. A screening test every 2 years was a safe and cost-effective strategy.

60-year results: annual examination with mydriatic fundus photography for groups 1,2 and 3 might save from 303 to 319 , from 58 to 62 and from 19 to 21 sight years, respectively. The results suggest that screening annually compared with 6 monthly was favoured.

For both types of patients, the mobile camera (Policy 2) had the lowest costs at $£ 449200$ per year and a cost per sight year saved of $f 2842$. Policy 2 was more cost-effective than policy 1 as long as the screening sensitivity and compliance were relatively high.

Results suggested there is little difference in the number of sight years saved between the different modes of screening when screening intervals are $\leq 1$ year and compliance is high.

Study perspective: Government Time horizon: Lifetime Discount rate: $5 \%$

Currency/price year: US\$ in 1986 prices
Outcomes: Person years of sight saved

Costs: Screening (eye

examination, angiography) and treatment (laser pan retinal or
All strategies resulted in cost savings. There is an economic advantage in adding semiannual visits under strategy 3 . Although it was slightly less cost-saving than annual 
Table 2 Continued

\begin{tabular}{|c|c|c|c|c|c|c|}
\hline $\begin{array}{l}\text { Author } \\
\text { (year) }\end{array}$ & $\begin{array}{l}\text { Type of economic } \\
\text { evaluation and model }\end{array}$ & Population studied & Comparators & $\begin{array}{l}\text { Methods (perspective, time } \\
\text { horizon and discount rate) }\end{array}$ & $\begin{array}{l}\text { Methods (costs, outcomes, } \\
\text { ICER and sensitivity } \\
\text { analyses) }\end{array}$ & Results and main conclusions \\
\hline & & & $\begin{array}{l}\text { and examination every } 6 \text { months } \\
\text { for those with DR } \\
\text { 4. full fundus photographs annually } \\
\text { 5. full fundus photographs annually } \\
\text { for patients with no DR and } \\
\text { examination every } 6 \text { months for } \\
\text { those with DR }\end{array}$ & & $\begin{array}{l}\text { focal) } \\
\text { ICER: None stated } \\
\text { Sensitivity analyses: One-way }\end{array}$ & $\begin{array}{l}\text { examination alone, more years of sight are saved } \\
\text { than less frequent examination. }\end{array}$ \\
\hline $\begin{array}{l}\text { Javitt et al } \\
(1994)^{6}\end{array}$ & $\begin{array}{l}\text { EE: CEA } \\
\text { Model: Simulation model } \\
\text { using PROPHET }\end{array}$ & $\begin{array}{l}\text { Hypothetical cohort of } \\
\text { patients with T2DM with DR }\end{array}$ & $\begin{array}{l}\text { Eight screening strategies: } \\
\text { (1) and (2) screening every } 2 \text { years. } \\
\text { Patients with background or more } \\
\text { advanced DR seen semiannually under } \\
\text { strategy } 1 \text { or annually under } \\
\text { strategy } 2 \text {. } \\
\text { (3), (4) and (5) screening every } \\
3 \text { years. Patients with background DR } \\
\text { scheduled every } 6 \text { months, } 12 \text { months } \\
\text { or } 18 \text { months, respectively } \\
\text { (6), (7) and (8) screening every } 4 \text { years } \\
\text { Patients with background DR } \\
\text { scheduled every } 6 \text { months, } 12 \text { months } \\
\text { or } 24 \text { months, respectively }\end{array}$ & $\begin{array}{l}\text { Study perspective: Government } \\
\text { Time horizon: Lifetime } \\
\text { Discount rate: } 5 \% \text { (varied } \\
\text { between } 2.5 \% \text { and } 10 \% \text { ) } \\
\text { Currency/price year: US\$ in } \\
1990 \text { prices }\end{array}$ & $\begin{array}{l}\text { Outcomes: Person years of } \\
\text { sight saved } \\
\text { Costs: Screening and treatment } \\
\text { and cost of blindness } \\
\text { ICER: None stated } \\
\text { Sensitivity analyses: One-way }\end{array}$ & $\begin{array}{l}\text { Changing the frequency of screening for patients } \\
\text { with no or mild background DR from } 1 \text { year to } \\
2 \text { years has no detrimental effect on years of } \\
\text { sight saved while reducing costs. } \\
\text { Once patients develop moderate non-proliferative } \\
\text { or more advanced DR, savings in sight-years are } \\
\text { sensitive to the screening interval. }\end{array}$ \\
\hline $\begin{array}{l}\text { Rein et al } \\
(2011)^{46}\end{array}$ & $\begin{array}{l}\text { EE: CUA } \\
\text { Model: Monte Carlo } \\
\text { simulation }\end{array}$ & $\begin{array}{l}\text { Hypothetical } 10 \text { million } \\
\text { patients with T2DM with no } \\
\text { or early DR }\end{array}$ & $\begin{array}{l}\text { Four screening methods: } \\
\text { 1. patient self-referral following visual } \\
\text { symptoms } \\
\text { 2. annual eye evaluation, } \\
\text { 3. biennial eye evaluation } \\
\text { 4. annual telemedicine screening in } \\
\text { primary care settings }\end{array}$ & $\begin{array}{l}\text { Study perspective: Societal } \\
\text { Time horizon: Lifetime } \\
\text { Discount rate: } 3 \% \\
\text { Currency/price year: US\$ in } \\
2010 \text { prices }\end{array}$ & $\begin{array}{l}\text { Outcomes: QALYs } \\
\text { Costs: Intervention (including } \\
\text { telemedicine) and treatment } \\
\text { costs and productivity losses } \\
\text { ICER: Cost per QALY gained } \\
\text { Sensitivity analyses: } \\
\text { Probabilistic }\end{array}$ & $\begin{array}{l}\text { Current annual eye evaluation was costly } \\
\text { compared with either treatment alternative. } \\
\text { Self-referral offered the lowest costs and QALYs, } \\
\text { followed by telemedicine, biennial evaluation and } \\
\text { annual evaluation. }\end{array}$ \\
\hline $\begin{array}{l}\text { Tung et al } \\
(2008)^{8}\end{array}$ & $\begin{array}{l}\text { EE: CEA and CUA } \\
\text { Model: Markov-decision } \\
\text { type model }\end{array}$ & $\begin{array}{l}\text { Community-based patients } \\
\text { with T2DM }\end{array}$ & $\begin{array}{l}\text { Five screening strategies compared } \\
\text { with no screening: } \\
\text { 1. annual screening } \\
\text { 2. biennial screening } \\
\text { 3. 3-year screening } \\
\text { 4. 4-year screening 5-year screening }\end{array}$ & $\begin{array}{l}\text { Study perspective: Not stated } \\
\text { Time horizon: } 10 \text { years } \\
\text { Discount rate: } 5 \% \\
\text { Currency/price year: New } \\
\text { Taiwan (NT) \$ in } 2004 \text { prices }\end{array}$ & $\begin{array}{l}\text { Outcomes: Sight years saved } \\
\text { and QALYs } \\
\text { Costs: Direct costs of screening, } \\
\text { drugs and treatment (laser } \\
\text { photocoagulation and surgery) } \\
\text { ICER: Cost per sight year saved } \\
\text { and cost per QALY gained } \\
\text { Sensitivity analyses: One-way }\end{array}$ & Annual screening should be conducted. \\
\hline $\begin{array}{l}\text { Vijan et al } \\
(2000)^{47}\end{array}$ & $\begin{array}{l}\text { EE: CUA } \\
\text { Model: Markov model. }\end{array}$ & Hypothetical T2DM patients & $\begin{array}{l}\text { Four screening strategies compared } \\
\text { with no screening: } \\
\text { 1. annual screening } \\
\text { 2. biennial screening } \\
\text { 3. } 3 \text {-year screening } \\
\text { 4. } 5 \text {-year screening }\end{array}$ & $\begin{array}{l}\text { Study perspective: Third party } \\
\text { payer (government and } \\
\text { societal used in sensitivity } \\
\text { analyses) } \\
\text { Time horizon: Lifetime } \\
\text { Discount rate: } 3 \% \\
\text { Currency/price year: US\$-year } \\
\text { not stated }\end{array}$ & $\begin{array}{l}\text { Outcomes: QALYs } \\
\text { Costs: Screening, } \\
\text { ophthalmology visits, laser } \\
\text { treatment and angiogram } \\
\text { ICER: Cost per QALY gained } \\
\text { Sensitivity analyses: One-way } \\
\text { and multivariate }\end{array}$ & $\begin{array}{l}\text { Screening every other year maybe the most } \\
\text { cost-effective option. with the option of tailoring } \\
\text { screening to the needs of different individuals. }\end{array}$ \\
\hline
\end{tabular}


every 2 years remained cost-effective. ${ }^{46}$ For those who already have retinopathy a greater degree of surveillance would be required, but results were mixed, depending on models used and assumptions made. Once retinopathy was detected, the screening interval should be 6 months; ${ }^{44} 45$ in contrast, two studies concluded that annual screening should be used; ${ }^{943}$ and the final study found that the most cost-effective option was to carry out two screening tests at 30 -month intervals. ${ }^{41}$

\section{DISCUSSION}

\section{Principal findings}

Studies broadly supported extending screening intervals beyond 1 year for patients with T2DM at low risk of progression to DR, such as those with well controlled diabetes on dietary treatment, with low HbA1c and no background retinopathy. Inevitably the lack of comparators in the evidence base introduces significant bias including selection bias and attrition bias-with those at least risk most likely to participate and those at most risk most likely to drop out. Both of these problems bias findings, that is, screening every 2 years will appear to be more beneficial and less risky than in fact it is. Other problems in the studies identified included heterogeneity in screening methods, in grading protocols, in defining risk, and uptake. Cost-effectiveness findings were conflicting. Three studies ${ }^{9} 44 \quad 43$ concluded that annual screening remains the most cost-effective. Risk stratification models show promise in providing tailored screening intervals based on individual risk factors, but none have been externally validated.

\section{Strengths and weaknesses}

This review is systematic with a priori study eligibility criteria and rigorous methods in selection, extraction, quality appraisal and synthesis of evidence. We were unable to pool evidence due to heterogeneity, and to assess publication bias. A thorough quality assessment was undertaken using recognised checklists.

None of the included studies allowed for direct comparison of different screening intervals in relation to the incidence of retinopathy or vision loss. Economic analyses were based on hypothetical simulation models. Strengths of the evidence base include: good reporting, real-world setting, ${ }^{1} \begin{array}{llll}26 & 32 & 34-36 & \text { large }\end{array}$ sample size, ${ }^{1} 263940$ adequate methods of participant recruitment and sufficient follow-up. ${ }^{26} 2730333742$ There were some notable limitations: high attrition, ${ }^{1} 253536$ systematic differences between attendees and non-attendees and substantial heterogeneity between studies, making it difficult to compare results with regard to the occurrence or progression of retinopathy. Therefore patients with 'no existing background retinopathy' should be interpreted as 'patients in whom no evidence for background retinopathy has been found'. ${ }^{18}$ There were also difficulties in measuring other risk factors such as duration of diabetes. There may be limited applicability to adults with T1DM as only a small proportion of the studies covered this group. While we did not find sufficiently robust evidence to suggest that the screening interval could safely be extended beyond 1 year, it should be noted that equally we did not find persuasive evidence that it should not be extended. Only one risk prediction algorithm was externally validated and this was on a Danish data $\operatorname{set}^{37}$ where there was a large amount of missing data, and where screening intervals are already stratified by risk so not applicable to a system such as the UK where uniform screening intervals currently pertain.

Cost-effectiveness models were of considerable complexity and included various inputs such as: progression rates between disease stages, interval between screening visits, compliance and sensitivity, and specificity of testing. In general, the models assumed equal treatment success irrespective of screening interval; most also assumed the same compliance rate and uncertainty in patient behaviour and compliance were not adequately included (eg, differential compliance with different screening intervals $\left.{ }^{49}\right)$. Individual patient characteristics which potentially determine optimal screening interval and the practicalities of providing individualised screening intervals, were not included. Most studies did include benefits of detecting other eye disorders. The clinical outcomes and methodologies of the models were heterogeneous and precluded meaningful synthesis in meta-analysis. Some studies did not assign utility scores to differing degrees of sight loss and models used averaged progression rates obtained from studies such as the UK Prospective Diabetes Study and the Wisconsin Epidemiologic Study of Diabetic Retinopathy, in which most patients do not progress quickly. Discrete Event Simulation models use such averages which are applied to a variety of patients whose risk of progression depends on their baseline characteristics. If this is the case, it may mean that individuals who progress at the fastest rate may not be effectively captured within the model; it is these individuals who would be most likely to influence any difference in outcomes between programmes. Three studies ${ }^{64} 45$ are over 20 years old, and their validity is questionable in light of medical advances, changing prevalence and costs.

\section{Comparison of findings with other reviews}

Three previous systematic reviews have been published. ${ }^{20} 4950$ The most recent of these ${ }^{20}$ did not identify six of the papers we have presented in this paper including two of the largest cohorts with $14554^{39}$ and $155114^{40}$ patients with diabetes. For one study $^{43}$ authors have misinterpreted biannual (every 6 months) as biennial (every 24 months). Echouffo-Tcheugui ${ }^{20}$ concluded that the screening interval could safely be extended to 2 years. Jones et $a l^{49}$ had broad aims and examined all aspects of costeffectiveness of DR screening. The Wessex Institute report ${ }^{50}$ also reviewed cost-effectiveness concluding that the limited evidence base suggests that more patients may lose their sight with a 2 year interval.

\section{Recommendations for future research}

Further research is needed on:

- how 'low-risk' patients should be identified,

- how different screening and grading protocols affect performance, and

- how extending the screening interval might affect uptake.

- An RCT randomising either individual patients or whole screening centres to a longer interval would provide robust data upon which to base policy decisions and underpin a rigorous cost-effectiveness analysis

- Risk stratification algorithms identified in this review showed considerable promise for optimising services and minimising costs, although the cost-effectiveness of such a strategy would need to be carefully considered, and risk algorithms validated.

\section{Implications for clinicians and policymakers}

Based on the strength of the evidence identified in this review, we cannot reliably predict the outcome of a change in screening intervals. While the invited interval might be 2 years, in practice with lower uptake the screening interval might extend well beyond this for some patients. Additionally, there would need to be a reliable and uniform method for identifying and recording risk of progression to STDR. Previous retinopathy screening 
results allow for risk stratification. This is a powerful predictor of risk, but is not currently measured consistently across the UK. ${ }^{18}$ Detection and treatment for diabetes has improved in recent years, with diabetes more likely to be diagnosed and treated. This in turn will reduce the risk of progression of DR.

There is a broader question about the level of evidence required to make changes to an existing screening programme. While the best evidence would be from RCTs or meta-analysis of several trials, this is not a practical or affordable approach for every decision on a screening programme. Most often after implementation, the screening programme will be extended, either in frequency or in the eligible population, a phenomenon known as 'mission creep'. Here, we considered the minimum level of evidence to be a two arm randomised or non-randomised trial investigating the effect of a 1-year interval vs a 2 -year interval on retinopathy rates and uptake, with appropriate costeffectiveness analysis. This was not available in the literature.

\section{CONCLUSION}

Observational and economic modelling studies in low-risk patients show little difference in clinical outcomes between screening intervals of 1 year or 2 years. The lack of experimental research design and heterogeneity in definition of those at low risk limits the reliability and validity of this conclusion. Cost-effectiveness literature provides mixed results. While we did not find sufficiently robust evidence to suggest that the screening interval could safely be extended beyond 1 year, it should be noted that equally we did not find persuasive evidence that it should not be extended. However we consider that current evidence does not support a move to extend the screening interval beyond 1 year.

Contributors RL performed the searches. RL and DT conducted the screening, data extraction and quality assessment for the clinical papers. HM led the data extraction and quality assessment for the cost-effectiveness papers. ST-P reviewed the risk stratification algorithms and drafted the paper. All authors were involved in reviewing, commenting, and editing drafts of the manuscript and all approved of the final manuscript and take responsibility for the integrity of the data and the accuracy of the data analysis. ST-P is guarantor.

Funding This work was supported by The Four Nations diabetic retinopathy steering group on behalf of the UK National Screening Committee, part of Public Health England. The funders provided feedback on the protocol and the draft report, but all decisions regarding study design; collection, analysis, and interpretation of data; the writing of the report; and submission for publication were taken independently by the study team. ST-P is supported by a postdoctoral fellowship from the National Institute for Health Research (NIHR). AC is supported by the NIHR Collaboration for Leadership in Applied Health Research and Care West Midlands at University Hospitals Birmingham NHS Foundation Trust. The views expressed are those of the authors and not necessarily those of the NHS, the NIHR or the Department of Health.

\section{Competing interests None.}

Provenance and peer review Not commissioned; externally peer reviewed.

Open Access This is an Open Access article distributed in accordance with the Creative Commons Attribution Non Commercial (CC BY-NC 4.0) license, which permits others to distribute, remix, adapt, build upon this work non-commercially, and license their derivative works on different terms, provided the original work is properly cited and the use is non-commercial. See: http://creativecommons.org/ licenses/by-nc/4.0/

\section{REFERENCES}

1 Thomas RL, Dunstan F, Luzio SD, et al. Incidence of diabetic retinopathy in people with type 2 diabetes mellitus attending the Diabetic Retinopathy Screening Service for Wales: retrospective analysis. BMJ 2012;344:e874.

2 Saadine JB, Fong DS, Yao J. Factors associated with follow-up eye examinations among persons with diabetes. Retina 2008;28:195-200.

3 Scanlon PH. The English national screening programme for sight-threatening diabetic retinopathy. J Med Screen 2008;15:1-4.
4 Agardh E, Agardh CD, Hansson-Lundblad C. The five-year incidence of blindness after introducing a screening programme for early detection of treatable diabetic retinopathy. Diabet Med 1993;10:555-9.

5 Stefansson E, Bek T, Porta M, et al. Screening and prevention of diabetic blindness. Acta Ophthalmol Scand 2000;78:374-85.

6 Javitt JC, Aiello LP, Chiang Y, et al. Preventive eye care in people with diabetes is cost-saving to the federal government: implications for health-care reform. Diabetes Care 1994;17:909-17.

7 Backlund LB, Algvere PV, Rosenqvist U. New blindness in diabetes reduced by more than one-third in Stockholm County. Diabet Med 1997;14:732-40.

8 Cheung N, Mitchell P, Wong TY. Diabetic retinopathy. Lancet 2010;376:124-36.

9 Tung $\mathrm{TH}$, Shih $\mathrm{HC}$, Chen SJ, et al. Economic evaluation of screening for diabetic retinopathy among Chinese type 2 diabetics: a community-based study in Kinmen Taiwan. J Epidemiol 2008;18:225-33.

10 Scanlon PH, Malhotra R, Greenwood RH, et al. Comparison of two reference standards in validating two field mydriatic digital photography as a method of screening for diabetic retinopathy. Br J Ophthalmol 2003;87:1258-63.

11 Olson JA, Strachan FM, Hipwell JH, et al. A comparative evaluation of digital imaging, retinal photography and optometrist examination in screening for diabetic retinopathy. Diabet Med 2003;20:528-34.

12 Mitchell P, Foran S, Wong TY, et al. Guidelines for the Management of Diabetic Retinopathy. Australia: Australian Diabetes Society for the National Health \& Medical Research Council, 2008. Contract No: 4176

13 Stefánsson E. Diabetic retinopathy screening. Pediatrics 2006;117:586

14 Socialstyrelsen. National guidelines for diabetes. Stockholm, Sweden, Socialstyrelsen. 2010. http://wwwsocialstyrelsense (accessed May 2011)

15 Hutchinson A, McIntosh A, Peters J, et al. Clinical guidelines and evidence review for Type 2 diabetes: diabetic retinopathy: early management and screening. Sheffield: ScHARR, University of Sheffield, 2001.

16 Diabetic Retinopathy Summary Benchmark-2013. American Academy of Opthalmology, 2013

17 Ting $\mathrm{D}, \mathrm{Ng}$ J, Morlet $\mathrm{N}$, et al. Diabetic retinopathy-screening and management by Australian GPs. Aust Fam Physician 2011;40:233-8.

18 Stratton IM, Aldington SJ. Risk stratification for diabetic eye screening. Diabetologia 2014;57:259.

19 McGhee S, Harding SP, Wong D. Individual risk assessment and information technology to optimise screening frequency for diabetic retinopathy by Aspelund et al(2011) Diabetologia 54:2525-2532. Graefes Arch Clin Exp Ophthalmol 2012;250:477-8

20 Echouffo-Tcheugui JB, Ali MK, Roglic G, et al. Screening intervals for diabetic retinopathy and incidence of visual loss: a systematic review. Diabet Med 2013;30:1272-92.

21 Husereau $D$, Drummond $M$, Petrou $S$, et al. Consolidated health economic evaluation reporting standards (CHEERS) statement. BMC Med 2013;11:80.

22 Philips Z, Bojke L, Sculpher M, et al. Good practice guidelines for decision-analytic modelling in health technology assessment. Pharmacoeconomics 2006;24:355-71.

23 Unit PHR. The critical skills appraisal programme: making sense of evidence. England: Public Health Resource Unit, 2006. http://www.casp-uk.net/

24 Steyerberg EW, Moons KG, van der Windt DA, et al. Prognosis research strategy (PROGRESS) 3: prognostic model research. PLOS Med 2013;10:e1001381.

25 Misra A, Bachmann M, Greenwood R, et al. Trends in yield and effects of screening intervals during 17 years of a large UK community-based diabetic retinopathy screening programme. Diabet Med 2009;26:1040-7.

26 Jones $\mathrm{CD}$, Greenwood RH, Misra A, et al. Incidence and progression of diabetic retinopathy during 17 years of a population-based screening program in England. Diabetes Care 2012;35:592-6.

27 Mehlsen J, Erlandsen M, Poulsen PL, et al. Identification of independent risk factors for the development of diabetic retinopathy requiring treatment. Acta Ophthalmol 2011;89:515-21.

28 Mehlsen J, Erlandsen M, Poulsen PL, et al. Individualized optimization of the screening interval for diabetic retinopathy: A new model. Acta Ophthalmol 2012;90:109-14

29 Kristinsson JK, Gudmundsson JR, Stefansson E, et al. Screening for diabetic retinopathy. Initiation and frequency. Acta Ophthalmol Scand 1995;73:525-8.

30 Olafsdóttir E, Stefánsson E. Biennial eye screening in patients with diabetes without retinopathy: 10-year experience. Br J Ophthalmol 2007;91:1599-601.

31 Soto-Pedre E, Hernaez-Ortega MC, Vazquez JA. Six-year retrospective follow-up study of safe screening intervals for sight-threatening retinopathy in patients with diabetes mellitus. J Diabetes Sci Technol 2009;3:812-18.

32 Agardh E, Tababat-Khani P. Adopting 3-year screening intervals for sight-threatening retinal vascular lesions in type 2 diabetic subjects without retinopathy. Diabetes Care 2011;34:1318-9.

33 Kohner EM, Stratton IM, Aldington SJ, et al. Relationship between the severity of retinopathy and progression to photocoagulation in patients with Type 2 diabetes mellitus in the UKPDS (UKPDS 52). Diabet Med 2001;18:178-84.

34 Maguire A, Chan A, Cusumano J, et al. The case for biennial retinopathy screening in children and adolescents. Diabetes Care 2005;28:509-13. 
35 Younis N, Broadbent DM, Harding SP, et al. Incidence of sight-threatening retinopathy in Type 1 diabetes in a systematic screening programme. Diabet Med 2003;20:758-65.

36 Younis N, Broadbent DM, Vora JP, et al. Incidence of sight-threatening retinopathy in patients with type 2 diabetes in the Liverpool Diabetic Eye Study: A cohort study. Lancet 2003;361:195-200.

37 Aspelund $\mathrm{T}$, Thornorisdottir $\mathrm{O}$, Olafsdottir $\mathrm{E}$, et al. Individual risk assessment and information technology to optimise screening frequency for diabetic retinopathy. Diabetologia 2011;54:2525-32.

38 Semeraro F, Parrinello G, Cancarini A, et al. Predicting the risk of diabetic retinopathy in type 2 diabetic patients. J Diabetes Complications 2011:25:292-7.

39 Stratton IM, Aldington SJ, Taylor DJ, et al. A simple risk stratification for time to development of sight-threatening diabetic retinopathy. Diabetes Care 2013;36:580-5.

40 Looker HC, Nyangoma SO, Cromie DT, et al. Predicted impact of extending the screening interval for diabetic retinopathy: the Scottish Diabetic Retinopathy Screening programme. Diabetologia 2013;56:1716-25.

41 Brailsford SC, Gutjahr WJ, Rauner MS, et al. Combined discrete-event simulation and ant colony optimisation approach for selecting optimal screening policies for diabetic retinopathy. Computational Management Science 2007:4:59-83.
42 Chalk D, Pitt M, Vaidya B, et al. Can the Retinal Screening Interval Be Safely Increased to 2 Years for Type 2 Diabetic Patients Without Retinopathy? Diabetes Care 2012;35:1663-8.

43 Dasbach EJ, Fryback DG, Newcomb PA, et al. Cost-effectiveness of strategies for detecting diabetic retinopathy (Structured abstract). Med Care 1991;29:20-39.

44 Davies R, Roderick P, Canning C, et al. The evaluation of screening policies for diabetic retinopathy using simulation. Diabet Med 2002;19:762-70.

45 Javitt J, Canner J, Frank $R$, et al. Detecting and treating retinopathy in patients with type I diabetes mellitus. A health policy model. Ophthalmology 1990;97:483-94; discussion 94-5.

46 Rein DB, Wittenborn JS, Zhang $X$, et al. The cost-effectiveness of three screening alternatives for people with diabetes with no or early diabetic retinopathy. Health Serv Res 2011;46:1534-61.

47 Vijan S, Hofer TP, Hayward RA. Cost-utility analysis of screening intervals for diabetic retinopathy in patients with type 2 diabetes mellitus. JAMA 2000;283:889-96.

48 Vickers AJ, Cronin AM. Everything you always wanted to know about evaluating prediction models (but were too afraid to ask). Urology 2010;76:1298-301.

49 Jones $S$, Edwards R. Diabetic retinopathy screening: a systematic review of the economic evidence. Diabet Med 2009;27:249-56.

50 Mellanby A, Milne R. Research WIfH, Development SD, Committee E. Reducing the interval for diabetic retinal screening. Wessex Institute for Health Research and Development, 1999. 